CARNETS DE Carnets de géographes

GÉOGRAPHES.

4 | 2012

Géographies critiques

\title{
Entretien Avec Bernard Bret
}

\section{Yann Calberac et Bernard Bret}

\section{OpenEdition}

Journals

Édition électronique

URL : http://journals.openedition.org/cdg/979

DOI : $10.4000 /$ cdg. 979

ISSN : 2107-7266

Éditeur

UMR 245 - CESSMA

Référence électronique

Yann Calberac et Bernard Bret, «Entretien Avec Bernard Bret », Carnets de géographes [En ligne], 4 |

2012, mis en ligne le 01 septembre 2012, consulté le 14 septembre 2020. URL : http://

journals.openedition.org/cdg/979

\section{(ब) $\Theta \Theta$}

La revue Carnets de géographes est mise à disposition selon les termes de la Licence Creative Commons Attribution - Pas d'Utilisation Commerciale - Pas de Modification 4.0 International. 


\section{ENTRETIEN AVEC BERNARD BRET}

Entretien réalisé le 22 août 2012 par Yann Calbérac, ATER en géographie, Université Paris-Sorbonne (IUFM de Paris), UMR 8185 ENeC. BERNARD BRET est Professeur émérite de géographie à l'Université de Lyon - Université Jean Moulin Lyon 3 (UMR 5600 Environnement, ville, société). Son contact : bernard.bret12@wanadoo.fr

\section{Est-ce que vous vous reconnaissez sous l'étiquette de "géographe critique " ou de "géographe radical " ?}

Je ne sais pas: c'est une question que je ne me suis jamais vraiment posée. Critique, sans doute, à l'égard de la géographie traditionnelle qui est celle que les gens de ma génération ont reçue durant leurs études dans la deuxième partie des années 1960, une géographie assez pauvre du point de vue de la théorisation. La géographie d'alors était très empirique et descriptive, avec les mérites des descriptions souvent bien faites (je ne dis pas que c'était sans intérêt aucun), mais elle ne décollait pas toujours de la description; et en l'absence d'une théorisation, elle ne permettait guère de comprendre réellement les phénomènes. L'enseignant qui, a contrario, m'a le plus marqué au cours des études, même si après j'ai pu diverger d'avec lui sur plusieurs points (mais c'est normal d'évoluer et de ne pas rester dépendant de la formation que I'on a reçue), c'est Yves Lacoste. J'ai une dette intellectuelle envers lui : c'est lui qui en 1965 a publié sa Géographie du sous-développement ${ }^{1}$. Aujourd'hui, l'ouvrage est bien dépassé, mais cela fait un demi-siècle qu'il a été écrit. A l'époque, c'était un ouvrage pionnier et c'est Yves Lacoste qui le premier m'a montré très clairement dans son enseignement que la géographie n'était pas une suite d'informations, mais un raisonnement sur les faits sociaux et sur leur inscription dans l'espace. Son enseignement détonnait par rapport à ce que faisaient ses collègues : chez certains, même si leurs cours étaient bien documentés et bien construits, on restait quand même sur sa faim pour ce qui est de l'explication des organisations territoriales, ce qui apparaît pourtant aujourd'hui comme le cœur de la discipline. Alors oui, je me sens critique par rapport à une géographie traditionnelle, mais une géographie traditionnelle qui est aujourd'hui dépassée depuis pas mal de décennies. Mais par rapport à la géographie qui se fait aujourd'hui, non, je ne suis pas critique. Je suis

\footnotetext{
${ }^{1}$ Lacoste Y. (1965) Géographie du sous-développement, Paris, PUF, 284 p. (NDLR : Toutes les notes sont de Yann Calbérac)
} 
simplement, comme tous les géographes, plus ou moins attiré par tel ou tel positionnement intellectuel.

Et par rapport à la société : les géographes critiques dénoncent les inégalités des sociétés et s'engagent pour les dénoncer, voire les réduire. Est-ce que cette appellation vous convient ? Est-ce que par vos travaux vous avez eu à cœur de dénoncer ces inégalités et de vous engager pour les résoudre?

Mes travaux se placent dans la sphère des sciences sociales : ils ont donc un objectif de connaissance. Comme pour toutes les sciences sociales, il faut chercher à comprendre les facteurs explicatifs des choses, à donner des explications correctes à partir d'hypothèses. Après, est-ce qu'on a aussi pour métier de transformer les choses ? La géographie, me semble-t-il, comporte les deux volets : c'est une discipline de connaissance dans le domaine des sciences sociales et une discipline qui relève de l'aménagement du territoire. Chacun peut avoir le positionnement qui correspond le mieux à ce qu'il sait faire ou aime faire. Personnellement, je me situe davantage sur le premier registre. Mais, si une analyse des sociétés et des territoires est bien faite je pense que cela a un impact sur la façon dont on peut ensuite aménager le territoire. Les deux dimensions se concilient alors elles-mêmes sans que ce soit nécessairement les mêmes personnes qui interviennent pour conduire les recherches et pour aménager.

Vous avez parlé du rôle qu'a joué Yves Lacoste dans votre formation intellectuelle. Est-ce que d'autres penseurs (géographes ou non) ont eu pour vous un rôle semblable à cette époque-là ?

Il y en a eu, mais celui qui a vraiment été décisif pour moi, dans ses pratiques d'enseignement et de recherche, c'est bien Yves Lacoste. Je pense aussi à Pierre George : je lui dois beaucoup et je ne veux absolument pas aujourd'hui, alors qu'il est décédé, dénigrer son apport. Toutefois, il faut bien reconnaître que son approche était assez décevante pour ce qui est de l'insertion des faits sociaux et politiques dans l'explication des organisations territoriales. Sans caricaturer ses engagements politiques et idéologiques, il est légitime d'exercer un esprit critique sur son héritage. Encore aujourd'hui, je m'interroge sur son enseignement. En 1968/69, il assurait le cours de préparation à l'agrégation sur I'URSS qui était au programme en géographie régionale. Son cours consistait en une présentation très classique - mais très claire, comme il savait le faire - des régions de l'Union soviétique. II dominait très bien son sujet. En revanche, de l'idée qui nous paraît essentielle aujourd'hui - à savoir que l'organisation de l'espace est en cohérence avec un type de société, et que la transformation sociale a une traduction spatiale - il ne disait mot. II parlait des combinats et de l'aménagement du territoire soviétique, mais en termes techniques et sans se demander si toutes ces opérations étaient menées en vue de construire une société nouvelle. A y réfléchir avec le recul, la mise en valeur de l'espace soviétique était célébrée, sans rien qui fût dit du coût humain de la chose, comme une volonté prométhéenne de dominer les lieux, un peu comme beaucoup avaient auparavant célébré la conquête de l'Ouest américain sans dénoncer le sort réservé aux Indiens. La géographie, en tant que science sociale, peut-elle se satisfaire d'un pareil discours ? Un point précis continue de me troubler sur la personnalité de Pierre George: il a présentait dans son cours - comme il l'avait fait dans son livre de la collection 
"Orbis ${ }^{2}$ - le système des canaux dit des cinq mers. Pourquoi ne disait-il pas que ces aménagements hydrauliques gigantesques avaient été faits par les prisonniers du Goulag ? Parmi les géographes, il a fallu attendre bien des années plus tard l'article de Roger Brunet pour éclairer l'esprit de tout le monde ${ }^{3}$. Il est tout de même étonnant de laisser sous silence des questions aussi graves et aussi décisives pour comprendre la logique globale du système : 1) en quoi cela correspond-il à un projet de société, 2) comment le projet a-t-il été mis en œuvre sous un régime tyrannique ? Dire que les réponses n'étaient pas données est insuffisant. La vérité est que les questions n'étaient même pas posées ! Or, Pierre George pouvait-il ignorer le rôle du travail forcé dans ces chantiers comme dans d'autres ? Sa pratique courante du russe et sa bonne connaissance de l'URSS conduisent à en douter. Comment expliquer son silence, alors même qu'il avait déjà pris ses distances avec le Parti Communiste ? Cela demeure pour moi une énigme et ne correspond ni à la rigueur scientifique que l'on doit avoir ni à la responsabilité intellectuelle que l'on doit assumer.

Continuons à dérouler le fil de votre parcours. Qu'est-ce qui vous a poussé, après l'agrégation à vous intéresser au Brésil ?

Si j'ai choisi la géographie, c'est peut-être naïf, c'est que je voulais travailler sur les problèmes de sous-développement pour reprendre le terme de l'époque. Au sortir du lycée, dans la répartition des sciences sociales que j'avais en tête, cette tâche incombait à la géographie. Aujourd'hui, je vois évidemment les choses autrement. Je reste convaincu que la géographie a son mot à dire dans l'affaire, mais je me tournerais plutôt vers l'économie, le droit, les sciences politiques et la philosophie. Mais à l'époque, la géographie me semblait la discipline la plus adéquate pour se saisir de ces phénomènes, et, pourquoi ne pas le dire, j'avais une attirance pour le métier d'enseignant. Mon idée, bien avant de choisir un sujet précis, était donc de travailler sur les problèmes de sous-développement ou de développement inégal comme on dirait plus volontiers aujourd'hui. Je voulais aussi étudier un pays susceptible de jouer un rôle important dans l'économie mondiale et qui paraissait avoir un potentiel important. Sur ce plan, le choix du Brésil s'est avéré exact. Pour des raisons que je ne saurais pas expliquer, avant même d'y avoir mis les pieds, j'avais une attirance pour le Nordeste qui m'apparaissait comme une interrogation (comment expliquer cette poche de semi-aridité dans l'intérieur?) largement nourrie du cinéma novo de l'époque. Mes motivations n'étaient qu'indirectement scientifiques !

\section{Avez-vous découvert sur place une autre manière de faire de la géographie, fondée sur d'autres références théoriques et méthodologiques?}

Comme tout chercheur qui doit apprendre du lieu qu'il prétend étudier, je suis redevable de beaucoup aux géographes brésiliens. Avec le souci de ne pas m'approprier les nombreux travaux des collègues brésiliens, j'ai plutôt cherché l'éclairage original que je pourrais éventuellement apporter. Dans la géographie brésilienne, il y a une tradition française qui s'explique notamment par la présence de Pierre Monbeig et de Claude Lévi-Strauss dans l'équipe qui a créé l'Université de Sao

\footnotetext{
${ }^{2}$ George P. (1962, $2^{\mathrm{e}}$ édition) L'URSS, Paris, PUF, $497 \mathrm{p}$.

3 Brunet R. (1981) "Géographie du Goulag ", L'espace géographique, n¹0-3, pp. 215-232. URL: http://www.persee.fr/web/revues/home/prescript/article/spgeo_0046-2497_1981_num_10_3_3657
} 
Paulo (la plus importante aujourd'hui). Il y a eu d'autres français à Rio de Janeiro et dans d'autres universités, tel, à Recife, Michel Rochefort qui ensuite a bien voulu diriger ma thèse d'Etat. De plus, beaucoup de géographes brésiliens ont fait leurs études supérieures et leur doctorat à Paris. L'héritage français reste donc important, mais il a été très concurrencé dans les années 1970 par l'influence nord-américaine. Ainsi a-t-on assisté à l'essor de la géographie quantitative (ce n'était ni dans mes compétences ni dans mon goût) : c'est un courant qui a eu une importance très lourde au Brésil, notamment à la fin des années 1960 et 1970 . Je pense notamment à un géographe comme Roberto Lobato Correa, de Rio de Janeiro. Pourquoi dans cette période la géographie quantitative se développe si vite au Brésil? On m’a fait remarquer (mais je ne sais pas ce que vaut cette explication) que cette " scientificité " plus grande permise par la mathématique donnait une apparence d'objectivité et de neutralité à une époque où la pensée était largement verrouillée par un régime dictatorial. Pour les géographes, l'approche quantitative aurait été une forme de liberté et de résistance. Je ne sais pas ce que vaut cette explication - elle ne m'a jamais vraiment convaincu - mais je l'ai entendue de la bouche de plusieurs collègues brésiliens. Ces deux influences - l'influence française et l'influence de la géographie quantitative nord-américaine - sont-elles encore importantes aujourd'hui ? Oui et non. La géographie quantitative a décliné car on en a vu les limites (Roberto Lobato Correa en est lui-même revenu: ce n'est pas grâce à ces outils que l'on peut vraiment comprendre les faits sociaux et l'organisation des lieux), et la tendance française s'est épuisée - comme en France - sous sa forme traditionnelle. Mais, sous une forme nouvelle, l'influence des géographes français reste forte avec des figures comme Paul Claval, Martine Droulers et Hervé Théry.

Les géographes brésiliens $\mathrm{m}^{\prime}$ ont beaucoup apporté. Je pense notamment à un géographe du Nordeste, Manuel Correia de Andrade (Université Fédérale du Pernambouc, à Recife) qui a travaillé sur les milieux ruraux et les structures agraires de la région. Il s'inscrivait dans la géographie traditionnelle: une géographie plutôt descriptive, avec une utilisation modérée de la statistique. II observait les phénomènes sur place, se revendiquait du terrain et décrivait les formes d'occupation du sol et les structures agraires : à qui appartient la terre ? Quelles sont les relations entre ceux qui possèdent la terre et ceux qui la travaillent ? Comment comprendre les violences et les revendications des populations sans terre ?... C'est une géographie assez traditionnelle dans sa façon d'exercer le métier (on va sur place et on rend compte de ce que l'on observe), mais très lucide sur l'organisation sociale: dans les ouvrages de Manuel Correia de Andrade, et notamment dans celui qui me paraît central, A terra e o homem no Nordeste $(1963)^{4}$, il y a une analyse critique très forte de l'injustice sociale qui sévit dans les campagnes du Nordeste, et l'inégalité des conditions est bien identifiée comme la cause principale de la pauvreté : la concentration foncière, les contrastes entre le latifundio et le microfundio, les paysans sans terre, les différentes formes que prend ce contraste entre celui qui a tout et celui qui n'a rien dans le contexte d'une économie de plantation littorale et dans l'économie de l'élevage du Sertao semi-aride.

\section{Quelles étaient les références théoriques, méthodologiques et idéologiques de ces géographes brésiliens ?}

\footnotetext{
${ }^{4}$ Correia de Andrade M. (1963) A terra e o homem no Nordeste, Sao Paulo, Editora Brasiliense, 265 p.
} 
Il y a - mais ce n'était pas le cas de Manuel Correia de Andrade - des géographes influencés par la pensée marxiste, et notamment, s'agissant de la géographie urbaine, par l'œuvre d'Henri Lefebvre. Parmi les auteurs qui ont été marquants pour m'aider à comprendre le Brésil, il y a eu un économiste pour lequel j'ai la plus grande admiration : Celso Furtado. II appartient à l'école de la dépendance. II était proche du sociologue Fernando Henrique Cardoso, qui, comme lui, a longtemps travaillé à Paris et qui devait ensuite être Président de la République de 1995 à 2003 . Revenons à Furtado: j'ai lu attentivement ses travaux. Il a écrit un ouvrage remarquable sur I'histoire économique du Brésil ${ }^{5}$ où il a expliqué les cycles économiques (les périodes pendant lesquelles il y avait un produit clé d'exportation - le sucre de canne, l'or, le café... - autour duquel s'organisent la société, l'économie et les territoires). C'était un universitaire qui avait un engagement politique fort : il a travaillé avec le président Kubitschek (celui qui a fondé Brasilia) et a créé la Surintendance pour le développement du Nordeste, la SUDENE, une agence de développement qui était un peu sur le modèle de la Cassa per il Mezzogiorno créée en Italie peu de temps auparavant pour prendre en main le développement du Midi italien. Furtado a été le premier surintendant de la SUDENE avant le coup d'Etat militaire de 1964 qui lui a valu son exil politique. Furtado a lancé des opérations de développement dans le Nordeste à travers un éventail de dispositions comparables à ce qui a été fait dans de nombreux pays : des incitations fiscales pour les investissements privés, un renforcement des investissements publics pour des infrastructures... Bref : une transformation profonde de la région qui reste malgré tout la poche de pauvreté du Brésil, dans un contexte d'économie de marché. Son idée était de consolider l'économie de marché en la rendant plus efficace économiquement et plus juste socialement, ce qui devait aboutir à un système plus stable politiquement car mieux accepté par les populations. Cela supposait évidemment des transformations sociales que le régime militaire (1964 1985) a empêchées. Avant de diriger la SUDENE, Furtado avait rédigé un rapport ${ }^{6}$ qui pose un diagnostic économique régional et ouvre des pistes d'action : c'est un rapport remarquable dont la lecture, plus d'un demi-siècle après sa rédaction, reste indispensable pour comprendre les blocages économiques du Nordeste. Quand Raymond Barre, futur Premier ministre, est allé à Bruxelles pour être commissaire européen, Celso Furtado a été élu sur la chaire laissée libre. Il a enseigné à I'Université Paris 1 pendant plusieurs années. C'était quelqu'un d'une intelligence remarquable et d'une rigoureuse intégrité politique : il n'a jamais transigé avec la dictature et il a été rappelé au gouvernement du Brésil après le retour de la démocratie. C'est lui qui a donné du Nordeste l'explication la plus convaincante, bien mieux que de nombreux géographes.

\section{Restons sur la question de l'engagement : comment avez-vous concilié (en France ou au Brésil) votre position d'enseignant-chercheur avec un éventuel engagement critique?}

\footnotetext{
${ }^{5}$ Furtado C. (1972) La formation économique du Brésil de l'époque coloniale aux temps modernes, Paris, La Haye, Mouton, 218 p. (édition brésilienne originale : 1959).

${ }^{6}$ Grupo de Trabalho para o Desenvolvimento Econômico do Nordeste (1959) Uma política de desenvolvimento econômico para o Nordeste, Rio de Janeiro, Imprensa Nacional, 94 p. La deuxième édition du rapport de 1967 est disponible en ligne : http://www.sudene.gov.br/acervo
} 
Dans mon cas personnel, le cas ne se posait pas vraiment: je suis allé au Brésil pour comprendre ce qui s'y passait, mais j'étais extérieur, et ma position d'étranger $m^{\prime}$ interdisait de $m^{\prime}$ engager directement. Mais vous posez un problème plus général : les rapports entre la préoccupation scientifique que l'on doit avoir comme chercheur et qu'il faut transmettre et un engagement plus direct dans la cité. Je pense bien sûr à l'ouvrage de Max Weber, Le savant et le politique ${ }^{7}$ : Weber met en garde contre le risque de faire d'un discours à prétention scientifique un discours partisan. II invite le savant à rester sur son registre de la connaissance et invite le politique à rester sur celui de la politique. C'est une préoccupation sans doute utile, mais qui a ses limites. Prenez le cas de Furtado: il a été un grand scientifique, mais il a aussi pris ses responsabilités politiques. Je ne dis pas qu'il mélangeait les deux choses, mais son engagement politique était clairement enraciné dans des convictions qui relevaient aussi de ses travaux scientifiques. La cloison n'est jamais totalement étanche. II faut donc être attentif à ceci : si on est sur le registre de la connaissance, il faut s'interdire toute facilité qui consisterait à forcer le réel pour servir une position politique. Ce serait détestable. D'ailleurs, rester sur le champ scientifique garantit que l'on soit crédible et que les idées produites méritent d'être débattues dans la cité et puissent servir éventuellement dans l'aménagement du territoire. Mais, bien sûr, être scientifique ne donne pas une légitimité autre que d'expertise. Dans le domaine politique, la légitimité vient de la démocratie.

Quand on parle aujourd'hui de géographies critiques, on pense au rôle fondateur des géographes critiques anglophones des années 1960 et 1970, comme David Harvey qui est aujourd'hui découvert en France. A quel moment et par quels canaux avezvous pris connaissance de ces travaux critiques ? Vous ont-ils été utiles ?

Je vais vous décevoir : je les ai découverts très tardivement et je ne les ai guère utilisés. $C^{\prime}$ est à travers l'anthologie coordonnée par Jean-François Staszak ${ }^{8}$ que j'ai découvert des auteurs que je connaissais pour certains à peine. C'est donc tardivement que j'ai découvert Harvey et d'autres géographes que je connais maintenant un peu mieux. Ce $\mathrm{n}^{\prime}$ est donc pas ces auteurs qui m'ont conduit à mener des recherches sur la production des inégalités. Si j'avais lu Harvey et d'autres plus tôt, sans doute aurais-je pensé autrement, mais c'est en lisant la Théorie de la justice ${ }^{9}$ du philosophe John Rawls que j'ai mis de l'ordre dans mes idées. J'ai vu dans son oeuvre une grille d'analyse pour comprendre les inégalités territoriales. John Rawls n'est pas géographe, il ne parle pas d'espace (le mot espace ne figure même pas une seule fois dans son livre), mais comme toute grande philosophie, sa pensée peut être utilisée de façon légitime par tout le monde. A partir de là, j'ai pu conduire une réflexion plus aboutie et plus intégrée sur les phénomènes territoriaux. Pourquoi Rawls? Dans la Théorie de la justice, il y a l'idée d'une justice sociale qui n'est pas l'égalitarisme, même si son principe premier reste la reconnaissance de la stricte égalité des personnes en ce qui concerne leur valeur intrinsèque (chacun a la même valeur d'existence). Mais comment organiser la vie sociale? Dans une démarche intellectuelle inscrite dans la tradition kantienne, Rawls développe l'idée qu'on ne peut pas concevoir une société

\footnotetext{
${ }^{7}$ Weber M. (1959) Le savant et le politique, Paris, Plon, 230 p. (édition originale allemande : 1919)

${ }^{8}$ Staszak, J.-F. (dir.) (2001) Géographies anglo-saxonnes, Tendances contemporaines, Paris, Belin, 313 p.

${ }^{9}$ Rawls J. (1971) A theory of justice, Cambridge, Harvard University Press, 607 p.
} 
strictement égalitariste dans la répartition des rôles parce que les personnes sont toutes différentes les unes des autres. Il y a des conditions réelles dans l'aptitude des personnes à occuper des positions sociales qui ne sont pas égales. II n'est donc guère possible de faire une société parfaitement égalitariste. On peut le regretter, mais ce n'est pas crédible. Ce qui est crédible en revanche, c'est d'optimiser les inégalités d'une façon qui valorise le plus possible ceux qui ont le moins (qu'il s'agisse des biens matériels, des revenus, des positions sociales...) : il faut alors trouver la configuration sociale la plus favorable aux plus démunis. La justice comme équité consiste en ce que la dynamique sociale tire le plus possible vers le haut celui qui est en bas. C'est le principe que Rawls appelle le maximin, la maximisation du minimum. Je tiens cette idée pour extrêmement féconde en géographie car elle énonce un principe qui peut être exploité dans l'analyse des différenciations spatiales : quelles sont les différences entre les lieux et comment les flux peuvent-ils réduire ou au contraire accroitre les inégalités, comment faire pour que les centres soient des pôles de développement (pour reprendre un terme de François Perroux), comment faire pour que l'accumulation qui s'y produit ne soit pas confisquée mais, par effet d'entraînement, serve à la périphérie ? Autant de questions qui me font conclure que le territoire socialisé peut être l'objet d'une approche rawlsienne.

Vous avez beaucoup travaillé dans le champ de la justice spatiale qui connaît une certaine vigueur aujourd'hui (je pense notamment à la revue JSSJ à laquelle vous collaborez) : quel est votre point de vue sur cette manière de mobiliser la justice spatiale pour alimenter une réflexion critique ? Je pense notamment à la diversité des thèmes et des échelles que ces études recouvrent aujourd'hui.

Votre question appelle de multiples réponses. Personnellement, j'ai fait un travail sur cette région très vaste qu'est le Nordeste brésilien car c'était le périmètre d'intervention de la SUDENE : pour comprendre les stratégies de développement, c'était l'échelle pertinente. Toutefois, pour comprendre cette région, il faut la resituer dans le contexte national: c'est une région périphérique de la région de Sao Paulo. Même si on prend un territoire délimité que l'on appelle région, il est impossible d'en comprendre les dynamiques si l'on reste à l'échelle régionale. Le contenu de l'analyse ne doit pas être limité à un territoire.

Sur l'idée de la justice spatiale dans la géographie, j'essaie, avec d'autres, de faire reconnaître ces thématiques dans la discipline. Mais il y a eu des travaux avant les miens : je pense notamment à Alain Reynaud et à son livre Société, espace et justice ${ }^{10}$ qui date de 1981 et dont on a republié quelques extraits dans la revue JSSJ pour célébrer le trentenaire de la publication. II a fait un travail pionnier d'une grande importance: c'est sous sa plume que l'on a, en français, la présentation la plus achevée du modèle centre/périphérie. Sur la justice distributive, l'apport d'Alain Reynaud est décisif, mais aujourd'hui, le livre paraît incomplet sur un point précis : il n'étudie pas les injustices qui ne résultent pas de l'accès aux biens matériels. Pour comprendre d'autres aspects de la justice, son apport doit être complété (comme pour la reconnaissance des identités et des minorités). La justice, c'est en effet davantage que la seule distribution des biens matériels. Aujourd'hui, Philippe Gervais-Lambony,

${ }^{10}$ Reynaud A. (1981) Société, espace et justice, Inégalités régionales et justice socio-spatiale, Paris, PUF, $263 \mathrm{p}$. 
professeur à Nanterre anime cette réflexion dans une démarche non seulement pluriscalaire, mais aussi plus large que celle développée par Alain Reynaud.

Pour finir, je voudrais connaître votre point de vue sur le foisonnement contemporain des approches critiques (et votre parcours que vous venez de retracer montre bien que l'intérêt pour la critique est une préoccupation ancienne) : que pensez-vous de cet essor?

Comme beaucoup d'autres, je le vois d'un très bon œil. C'est un signe de vitalité disciplinaire que d'avoir un regard critique sur ce qui a été fait. Surtout, et c'est selon moi le plus important, cette progression marque des préoccupations qui sont dans l'ordre de l'éthique. Cette préoccupation éthique doit être un élément constitutif du raisonnement. II ne s'agit pas de faire une géographie qui analyse les territoires d'une façon qui se voudrait neutre, puis, en conclusion, un peu comme une cerise sur un gâteau, de dire que tel processus est juste ou que tel autre ne l'est pas. Pourquoi cela ne va pas ? Cela peut paraître sympathique, mais cela me semble critiquable dans le fait que la conclusion ne reposerait pas sur une démarche scientifique et résulterait d'une sorte d'intuition de justice (sans doute respectable) dénuée de fondement pour la légitimer. II faut éviter cela. II faut au contraire avoir un raisonnement scientifique qui intègre la dimension éthique à l'analyse. C'est ce que permet à mon avis une approche rawlsienne parce que la théorie de John Rawls repose elle-même sur une assise purement rationnelle. Ce qui est fondamental, c'est que l'analyse territoriale s'appuie sur des bases théoriques qui intègrent le concept de justice. Tel est mon avis. Il existe bien sûr d'autres fondements théoriques que Rawls, et d'autres approches que les collègues de la revue JSSJ trouvent plus pertinentes (sans doute suis-je le seul rawlsien de l'équipe): cela enrichit la discussion et c'est tant mieux tant que la préoccupation éthique demeure au cœur du raisonnement.

Reste à éclaircir le mot de territoire utilisé ici à plusieurs reprises. Quand je parlais à l'instant d'un territoire périphérique - est-il tiré vers le haut par le centre ou vers le bas ? - il y a une certaine simplification qui pourrait alimenter un contre-sens. Parler d'un territoire pour examiner le sort des personnes qui y vivent exige de bien comprendre qu'elles n'ont pas toutes des intérêts convergents: des groupes sociaux peuvent avoir intérêt à ce que leur territoire soit mis en périphérie s'ils captent des ressources à leur profit dans leur rôle d'intermédiation avec le centre. Tous les territoires, qu'il s'agisse des centres ou des périphéries (à toutes les échelles), sont entre les mains de classes sociales aux intérêts divergents. Cela pose le gros problème des interventions sur les lieux : le risque, $c^{\prime}$ est que les discriminations positives à base territoriale (on aide une région caractérisée par des indicateurs économiques très bas, comme dans le Mezzogiorno italien ou le Nordeste brésilien) soient confisquées par la classe sociale dominante. Il est évident que certains groupes sociaux favorisés masquent leurs intérêts personnels ou de classe sous des revendications territoriales. Des individus qui ont de gros moyens financiers et qui ne sont sans doute pas à plaindre se lamentent sur le sort de leur région et demandent des aides ! Mais à qui va aller l'aide? A eux ou aux pauvres de la région ? Dans l'intention d'aider les plus démunis, attention à ne pas donner plus à ceux qui ont déjà davantage ! Ce qu'il faut, c'est dénoncer l'instrumentalisation du territoire par les groupes dominants et mettre en œuvre les mesures qui permettent effectivement de s'approcher du maximin. Parce 
Carnets de géographes, $\mathrm{n} 04$, septembre 2012

Rubrique Carnets de recherches

qu'elle s'occupe du socio-spatial, la géographie se doit d'apporter sa contribution dans cette affaire.

Merci.

Merci à vous! 